PROCEEDINGS OF THE

AMERICAN MATHEMATICAL SOCIETY

Volume 135, Number 5, May 2007, Pages 1249-1253

S 0002-9939(06)08634-5

Article electronically published on October 18, 2006

\title{
EQUIVARIANT CRYSTALLINE COHOMOLOGY AND BASE CHANGE
}

\author{
ELMAR GROSSE-KLÖNNE
}

(Communicated by Michael Stillman)

\begin{abstract}
Given a perfect field $k$ of characteristic $p>0$, a smooth proper $k$-scheme $Y$, a crystal $E$ on $Y$ relative to $W(k)$ and a finite group $G$ acting on $Y$ and $E$, we show that, viewed as a virtual $k[G]$-module, the reduction modulo $p$ of the crystalline cohomology of $E$ is the de Rham cohomology of $E$ modulo $p$. On the way we prove a base change theorem for the virtual $G$ representations associated with $G$-equivariant objects in the derived category of $W(k)$-modules.
\end{abstract}

\section{THE THEOREM}

Let $k$ be a perfect field of characteristic $p>0$, let $W$ denote its ring of Witt vectors, let $K=\operatorname{Quot}(W)$. Let $Y$ be a proper and smooth $k$-scheme and suppose that the finite group $G$ acts (from the right) on $Y$. Let $E$ be a locally free, finitely generated crystal of $\mathcal{O}_{Y / W}$-modules and suppose that for each $g \in G$ we are given an isomorphism of crystals $\tau_{g}: E \rightarrow g^{*} E$ (where $g^{*} E$ denotes the pullback of $E$ via $g: Y \rightarrow Y$ ) such that $g_{2}^{*}\left(\tau_{g_{1}}\right) \circ \tau_{g_{2}}=\tau_{g_{2} g_{1}}$ (equality as maps $\left.E \rightarrow\left(g_{2} g_{1}\right)^{*} E=g_{2}^{*} g_{1}^{*} E\right)$ for any two $g_{1}, g_{2} \in G$. For $s \in \mathbb{Z}$ let $H_{c r y s}^{s}(Y / W, E)$ denote the $s$-th crystalline cohomology group (relative to $\operatorname{Spf}(W)$ ) of the crystal $E$, a finitely generated $W$-module which is zero if $s \notin[0,2 \operatorname{dim}(Y)]$ (see [1]). On the other hand, the reduction modulo $p$ of the crystal $E$ is equivalent with a locally free $\mathcal{O}_{Y}$-module $E_{k}$ with connection $E_{k} \rightarrow \Omega_{Y}^{1} \otimes_{\mathcal{O}_{Y}} E_{k}$; here $\Omega_{Y}^{1}$ denotes the $\mathcal{O}_{Y^{-}}$ module of differentials of $Y / k$. Let $\Omega_{Y}^{\bullet} \otimes E_{k}$ denote the corresponding de Rham complex. The cohomology group $H^{s}\left(Y, \Omega_{Y}^{\bullet} \otimes E_{k}\right)$ is a finite-dimensional $k$-vector space which is zero if $s \notin[0,2 \operatorname{dim}(Y)]$. The isomorphisms $\tau_{g}$ for $g \in G$ provide each $H_{c r y s}^{s}(Y / W, E)$, each $H^{s}\left(Y, \Omega_{Y}^{\bullet} \otimes E_{k}\right)$ and each $H^{s}\left(Y, \Omega_{Y}^{t} \otimes E_{k}\right)$ for $t \geq 0$ with an action of $G$ (from the left). By definition, the reduction modulo $p$ of the $K[G]$ module $H_{\text {crys }}^{s}(Y / W, E) \otimes_{W} K$ is the $k[G]$-module obtained by reducing modulo $p$ the $G$-stable $W$-lattice $H_{c r y s}^{s}(Y / W, E) /\left(\right.$ torsion) in $H_{c r y s}^{s}(Y / W, E) \otimes_{W} K$.

Theorem 1.1. For any $j$, the following three virtual $k[G]$-modules are the same:

(i) the reduction modulo $p$ of the virtual $K[G]$-module

$$
\sum_{s}(-1)^{s} H_{\text {crys }}^{s}(Y / W, E) \otimes_{W} K
$$

Received by the editors February 15, 2005 and, in revised form, November 21, 2005. 2000 Mathematics Subject Classification. Primary 14F30, 13Dxx.

Key words and phrases. Crystalline cohomology, base change, virtual representation. 
(ii) $\sum_{s}(-1)^{s} H^{s}\left(Y, \Omega_{Y}^{\bullet} \otimes E_{k}\right)$;

(iii) $\sum_{s, t}(-1)^{s+t} H^{s}\left(Y, \Omega_{Y}^{t} \otimes E_{k}\right)$.

An obvious variant of Theorem 1.1 holds in logarithmic crystalline cohomology, for crystals $E$ on the logarithmic crystalline site of $Y / W$ with respect to a log structure defined by a normal crossings divisor on $Y$. Similarly, the proof which we give below also shows the analog of Theorem 1.1 for the $\ell$-adic cohomology $(\ell \neq p)$ of constructible $\ell$-adic sheaves on $Y$, even if $Y / k$ is not proper. Of course, the result in the $\ell$-adic case (even for nonproper $Y / k$ ) is well known; it has been used for investigating the reduction modulo $\ell$ of the Deligne-Lusztig characters of groups $G=\mathbb{G}(\mathbb{F})$, where $\mathbb{G}$ is a reductive group over a finite field $\mathbb{F}$ of characteristic $p$. In [3] we use the variant of Theorem 1.1 in logarithmic crystalline cohomology to show that these Deligne-Lusztig characters, usually defined via $\ell$-adic cohomology of certain $\mathbb{F}$-varieties which are nonproper in general, can also be expressed through the log crystalline cohomology of suitable log crystals on suitable proper and smooth $\mathbb{F}$-varieties with a normal crossings divisor. Unfortunately, the (more geometric) proof of the $\ell$-adic analog of Theorem 1.1 (due to Deligne and Lusztig; see for example 2, Lemma 12.4 and A3.15) breaks down for crystalline cohomology. On the other hand, our proof of Theorem 1.1 contains a result (Theorem 2.1) on Gactions on strictly perfect complexes in the derived category which should be of independent interest.

\section{THE PROOF}

Proof of Theorem 1.1. (ii)=(iii) is clear. By 1 we know that the total crystalline cohomology $\mathbb{R} \Gamma_{\text {crys }}(Y / W, E)$, as an object in the derived category $D(W)$ of the category of $W$-modules, is represented by a complex of $W$-modules of finite tordimension and with finitely generated cohomology; by functoriality, $G$ acts on $\mathbb{R} \Gamma_{\text {crys }}(Y / W, E)$. Also from [1] we know that the total crystalline cohomology commutes with base change, i.e. that $\mathbb{R} \Gamma_{\text {crys }}(Y / W, E) \otimes_{W}^{\mathbb{L}} k$ is the total crystalline cohomology of the reduction modulo $p$ of $E$ (as a crystal relative to $\operatorname{Spec}(k)$ ). But the latter is known (see [1, Corollary 7.4) to be the de Rham cohomology of $E_{k}$; i.e., its $s$-th cohomology group is $H^{s}\left(Y, \Omega_{Y}^{\bullet} \otimes E_{k}\right)$. Hence (i)=(ii) follows from Theorem 2.1 below.

Let $A$ be a complete discrete valuation ring with perfect residue field $k$ of characteristic $p>0$ and fraction field $K$ of characteristic 0 . Let $L^{\bullet}$ be a complex of $A$-modules of finite tor-dimension and with finitely generated cohomology; by [1], Lemma 7.15, this is equivalent with saying that $L^{\bullet}$ is quasi-isomorphic to a strictly perfect complex, i.e. a bounded complex of finitely generated projective $A$-modules. Suppose the finite group $G$ acts on $L^{\bullet}$ when $L^{\bullet}$ is viewed as an object in the derived category $D(A)$ of the category of $A$-modules. Then each cohomology group $H^{i}\left(L^{\bullet} \otimes_{A} K\right)=H^{i}\left(L^{\bullet}\right) \otimes_{A} K\left(\right.$ resp. each cohomology group $\left.H^{i}\left(L^{\bullet} \otimes_{A}^{\mathbb{L}} k\right)\right)$ becomes a representation of $G$ on a finite-dimensional $K$-vector space (resp. $k$-vector space).

Theorem 2.1. The virtual $k[G]$-module $\sum_{i}(-1)^{i} H^{i}\left(L^{\bullet} \otimes_{A}^{\mathbb{L}} k\right)$ is the reduction (modulo the maximal ideal of $A$ ) of the virtual $K[G]$-module $\sum_{i}(-1)^{i} H^{i}\left(L^{\bullet}\right) \otimes_{A} K$. Equivalently, the restriction of the character of $\sum_{i}(-1)^{i} H^{i}\left(L^{\bullet}\right) \otimes_{A} K$ to the subset of p-regular elements of $G$ is the Brauer character of $\sum_{i}(-1)^{i} H^{i}\left(L^{\bullet} \otimes_{A}^{\mathbb{L}} k\right)$.

We say that the automorphism $\gamma$ of the finitely generated $A$-module $M$ is prime to $p$ if and only if the following holds. For any finite extension $A^{\prime} \supset A$ with a discrete 
valuation ring $A^{\prime}$ and for any two $\gamma \otimes_{A} A^{\prime}$-stable submodules $N, N^{\prime}$ of $M \otimes_{A} A^{\prime}$ with $N^{\prime} \subset N$ and such that $N / N^{\prime}$ is a cyclic $A^{\prime}$-module, the endomorphism which $\gamma \otimes_{A} A^{\prime}$ induces on $N / N^{\prime}$ is of finite order prime to $p$.

Lemma 2.2. Let $\gamma$ be an automorphism of the finitely generated A-module $M$.

(a) If $M$ is free, then $\gamma$ is prime to $p$ if and only if the roots of the characteristic polynomial of $\gamma$ are roots of unity of order prime to $p$. In particular, $\left.\gamma\right|_{N}: N \rightarrow N$ is prime to $p$ for each submodule $N$ of $M$ with $\gamma(N)=N$.

(b) Let $M_{1} \subset M$ be a submodule with $\gamma\left(M_{1}\right)=M_{1}$ and such that $M_{2}=M / M_{1}$ is free. Let $\gamma_{1}$, resp. $\gamma_{2}$, be the induced automorphism of $M_{1}$, resp. of $M_{2}$. If $\gamma_{1}$ and $\gamma_{2}$ are prime to $p$, then $\gamma$ is prime to $p$.

Proof. Statement (a) is clear. (b) Let $N^{\prime} \subset N \subset M \otimes_{A} A^{\prime}$ be as in the definition. If $N \subset M_{1} \otimes_{A} A^{\prime}$ the hypothesis on $\gamma_{1}$ applies. Otherwise, since $M_{2} \otimes_{A} A^{\prime}$ is free over $A^{\prime}$ and $N / N^{\prime}$ is cyclic, $N / N^{\prime}$ maps injectively to $M_{2} \otimes_{A} A^{\prime}$ and the hypothesis on $\gamma_{2}$ applies.

Proof of Theorem 2.1. The problem is of course that the $H^{i}\left(L^{\bullet}\right)$ may have torsion, i.e., $H^{i}\left(L^{\bullet}\right) \otimes_{A} k \neq H^{i}\left(L^{\bullet} \otimes_{A}^{\mathbb{L}} k\right)$ in general. Similarly, the task would be easy if we knew that there is a strictly perfect complex $K^{\bullet}$ quasi-isomorphic to $L^{\bullet}$ such that the action of $G$ on $L^{\bullet}$ in $D(A)$ is given by the action of $G$ on $K^{\bullet}$ by true morphisms of complexes (not just by morphisms in $D(A)$ ). We introduce some notation. For an automorphism $\gamma: L^{\bullet} \rightarrow L^{\bullet}$ in $D(A)$ let $\epsilon_{1}^{i}, \ldots, \epsilon_{n(i)}^{i}\left(\right.$ with $\left.n(i)=\operatorname{dim}_{k} H^{i}\left(L^{\bullet} \otimes_{A}^{\mathbb{L}} k\right)\right)$ denote the roots of the characteristic polynomial of $\gamma$ acting on $H^{i}\left(L^{\bullet} \otimes_{A}^{\mathbb{L}} k\right)$ and let $\widetilde{\epsilon}_{1}^{i}, \ldots, \widetilde{\epsilon}_{n(i)}^{i}$ denote their Teichmüller liftings. On the other hand, let $\xi_{1}^{i}, \ldots, \xi_{n^{\prime}(i)}^{i}$ (with $n^{\prime}(i)=\operatorname{dim}_{K} H^{i}\left(L^{\bullet}\right) \otimes_{A} K$ ) denote the roots of the characteristic polynomial of $\gamma$ acting on $H^{i}\left(L^{\bullet}\right) \otimes_{A} K$. Then let

$$
\begin{gathered}
\operatorname{Br}\left(\gamma, H^{\odot}\left(L^{\bullet} \otimes_{A}^{\mathbb{L}} k\right)\right)=\sum_{i}(-1)^{i} \sum_{j=1}^{n(i)} \widetilde{\epsilon}_{j}^{i}, \\
\operatorname{Tr}\left(\gamma, H^{\odot}\left(L^{\bullet}\right) \otimes_{A} K\right)=\sum_{i}(-1)^{i} \sum_{j=1}^{n^{\prime}(i)} \xi_{j}^{i} .
\end{gathered}
$$

What we must show is that for all $p$-regular elements $g \in G$ (those whose order in $G$ is not divisible by $p$ ), if $\gamma: L^{\bullet} \rightarrow L^{\bullet}$ denotes the corresponding automorphism of $L^{\bullet}$ in $D(A)$, then

$$
\operatorname{Br}\left(\gamma, H^{\odot}\left(L^{\bullet} \otimes_{A}^{\mathbb{L}} k\right)\right)=\operatorname{Tr}\left(\gamma, H^{\odot}\left(L^{\bullet}\right) \otimes_{A} K\right) .
$$

Clearly it is enough to show the following statement. For any strictly perfect complex $L^{\bullet}$ of $A$-modules (not necessarily endowed with a $G$-action in $D(A)$ ) and for any automorphism $\gamma: L^{\bullet} \rightarrow L^{\bullet}$ in $D(A)$ which on the cohomology modules induces automorphisms prime to $p$ we have

$$
\operatorname{Br}\left(\gamma, H^{\odot}\left(L^{\bullet} \otimes_{A}^{\mathbb{L}} k\right)\right)=\operatorname{Tr}\left(\gamma, H^{\ominus}\left(L^{\bullet}\right) \otimes_{A} K\right) .
$$

We use induction on the minimal $m \in \mathbb{Z}_{\geq 0}$ with the following property: after a suitable degree shift we have $L^{i}=0$ for all $i \notin[0, m]$. For $m=0$ the statement is clear from Lemma 2.2(a). Now let $m \geq 1$; shifting degrees we may assume $L^{i}=0$ 
for all $i \notin[0, m]$. Let $d^{m}: L^{m-1} \rightarrow L^{m}$ denote the differential. Choose a sub- $k$ vector space $N_{k}^{m-1}$ of $L^{m-1} \otimes k$ which under $d^{m} \otimes k$ maps isomorphically to the kernel of

$$
L^{m} \otimes k \rightarrow H^{m}\left(L^{\bullet} \otimes k\right)=H^{m}\left(L^{\bullet}\right) \otimes k .
$$

Then $N_{k}^{m-1}=N^{m-1} \otimes k$ for a direct summand $N^{m-1}$ of $L^{m-1}$. By construction, $N^{m-1}$ maps isomorphically to its image $N^{m}$ in $L^{m}$. Thus, setting $N^{i}=0$ if $i \notin\{m-1, m\}$, the subcomplex $N^{\bullet}$ of $L^{\bullet}$ is acyclic. Dividing it out we may therefore assume $L^{m} \otimes k=H^{m}\left(L^{\bullet} \otimes k\right)$. Since the functor $K^{-}(\operatorname{proj}-A) \rightarrow D(A)$ from the homotopy category of complexes of projective $A$-modules bounded above to $D(A)$ is fully faithful, the action of $\gamma$ on $L^{\bullet}$ in $D(A)$ is in fact represented by a true morphism of complexes $\gamma^{\bullet}: L^{\bullet} \rightarrow L^{\bullet}$. Base changing to a finite extension of $A$ by a discrete valuation ring (this does not affect the numbers $B r$ and $T r$ ) we may suppose that the characteristic polynomial of $\gamma^{m}: L^{m} \rightarrow L^{m}$ splits in $A$ (we remark that $\gamma^{m}$ is bijective: this follows from $L^{m} \otimes k=H^{m}\left(L^{\bullet} \otimes k\right)$ and the fact that $\gamma$ acts bijectively on $\left.H^{m}\left(L^{\bullet} \otimes k\right)\right)$. We therefore find a $\gamma^{m}$-stable filtration

$$
(0)=F^{0} \subset F^{1} \subset \ldots \subset F^{s}=L^{m} \quad\left(s=\operatorname{rk}\left(L^{m}\right)\right)
$$

such that $G^{e}=F^{e} / F^{e-1}$ is free of rank one, for any $1 \leq e \leq s$. The cyclic $A$-module

$$
\frac{F^{e}}{\left(F^{e} \cap \operatorname{im}\left(d^{m}\right)\right)+F^{e-1}}
$$

is a $\gamma^{m}$-stable subquotient of $H^{m}\left(L^{\bullet}\right)$ (it is nonzero because of $L^{m} \otimes k=$ $\left.H^{m}\left(L^{\bullet} \otimes k\right)\right)$; hence $\gamma^{m}$ acts on it by multiplication with a root of unity of order prime to $p$. Let $\xi_{e} \in A^{\times}$denote its Teichmüller lifting. Choose $\ell_{e} \in F^{e}$ which represents a basis element of $G^{e}$; then $\ell_{1}, \ldots, \ell_{s}$ is a basis of $L^{m}$. Modulo $F^{e-1}$ the class of $\xi_{e} \ell_{e}-\gamma^{m}\left(\ell_{e}\right) \in F^{e}$ lies in $\operatorname{im}\left(d^{m}\right)$. Choose a $t_{e} \in L^{m-1}$ with

$$
d^{m}\left(t_{e}\right)=\xi_{e} \ell_{e}-\gamma^{m}\left(\ell_{e}\right) \text { modulo } F^{e-1} .
$$

Let $t: L^{m} \rightarrow L^{m-1}$ denote the $A$-linear map which sends $\ell_{e}$ to $t_{e}$, for each $1 \leq e \leq s$. Using $t$ we see that we may modify $\gamma^{\bullet}$ within its homotopy class to achieve that the filtration (11) is still $\gamma^{m}$-stable and such that $\gamma^{m}$ acts on each $G^{e}$ by multiplication with a root of unity of prime-to- $p$ order in $A^{\times}$. Therefore we may assume that $\gamma^{m}: L^{m} \rightarrow L^{m}$ is prime to $p$. Let $L_{1}^{m}=L^{m}$ and $L_{1}^{i}=0$ for $i \neq m$. Then $L_{1}^{\bullet}$ is a $\gamma^{\bullet}$-stable subcomplex of $L^{\bullet}$ and since $\operatorname{Br}(\gamma)$ and $\operatorname{Tr}(\gamma)$ are additive in exact $\gamma^{\bullet}$-equivariant sequences of complexes it suffices to show $\operatorname{Br}(\gamma)=\operatorname{Tr}(\gamma)$ for the complexes $L_{1}^{\bullet}$ and $L^{\bullet} / L_{1}^{\bullet}$. Since these complexes are shorter than $L^{\bullet}$ this follows from the induction hypothesis. Indeed, the prime-to- $p$ hypothesis is clearly satisfied for $L_{1}^{\bullet}$, so it remains to show that $\gamma^{\bullet}$ induces automorphisms prime to $p$ on the cohomology modules of $L^{\bullet} / L_{1}^{\bullet}$. In degrees smaller than $m-1$ this is clear from the corresponding hypothesis on $L^{\bullet}$; only $H^{m-1}\left(L^{\bullet} / L_{1}^{\bullet}\right)$ is critical. But $H^{m-1}\left(L^{\bullet}\right)$ is a submodule of $H^{m-1}\left(L^{\bullet} / L_{1}^{\bullet}\right)$ and the quotient

$$
Q=H^{m-1}\left(L^{\bullet} / L_{1}^{\bullet}\right) / H^{m-1}\left(L^{\bullet}\right)
$$

maps isomorphically to a submodule of $L_{1}^{m}=L^{m}$. By Lemma 2.2(b) it suffices to show that $\gamma^{\bullet}$ induces automorphisms prime to $p$ on $H^{m-1}\left(L^{\bullet}\right)$ and on $Q$. For $H^{m-1}\left(L^{\bullet}\right)$ this holds by hypothesis; for $Q$ this follows from Lemma 2.2(a). 


\section{REFERENCES}

[1] P. Berthelot, A. Ogus, Notes on crystalline cohomology. Princeton University Press (1978). MR0491705 (58:10908)

[2] M. Cabanes, M. Enguehard, Representation theory of finite reductive groups. New Mathematical Monographs, 1. Cambridge University Press, Cambridge (2004). MR 2057756|(2005g:20067)

[3] E. Grosse-Klönne, On the crystalline cohomology of Deligne-Lusztig varieties, to appear in Finite Fields and Their Applications.

Mathematisches Institut der Universität MÜnster, Einsteinstrasse 62, 48149 Münster, Germany

E-mail address: klonne@math.uni-muenster.de 